\title{
CERTAIN HADAMARD DESIGNS
}

\section{P. KESAVA MENON}

1. The object of this paper is to construct Hadamard designs for the parameters

$$
v=4 m^{2}-1, \quad k=2 m^{2}-1, \quad \lambda=m^{2}-1, \quad n=m^{2}
$$

where both $2 m+1$ and $2 m-1$ are prime powers.

2. Let $G F\left(p^{l}\right)$ be the Galois field of $p^{l}$ elements and let $x$ be a primitive element of the field. We shall denote the additive group of the field by $F$, the sets containing the odd and even powers of $x$ by $C_{\rho}, C_{\theta}$ respectively, and the set consisting of the single element $o$ by $C$ so that

$$
F=C+C_{\mathrm{o}}+C_{\mathrm{e}}
$$

If $A, B$ are two aggregates of elements of $F$ we shall denote by $A B$ the aggregate formed by adding each element of $A$ to every element of $B$. We shall also denote the aggregate obtained by taking $A a$ times by $a A$. Then we have the following

LEMMa 1. If $p^{l} \equiv 1(\bmod 4)$, then

$$
\begin{aligned}
C_{0} C_{0} & =\frac{p^{l}-1}{4}\left(C_{0}+C_{e}\right), \\
C_{0}^{2} & =\frac{p^{l}-1}{2} C+\frac{p^{l}-5}{4} C_{0}+\frac{p^{2}-1}{4} C_{0}, \\
C_{0}^{2} & =\frac{p^{l}-1}{2} C+\frac{p^{l}-1}{4} C_{0}+\frac{p^{l}-5}{4} C_{0} .
\end{aligned}
$$

Proof. The aggregate $C_{0} C_{e}$ consists of the elements

$$
x^{2 r-1}+x^{2 s} \quad(r, s=1,2, \cdots,(p-1) / 2) .
$$

If $x^{k}=x^{2 r-1}+x^{2 s}$ then $x^{k+i}=x^{2 r-1+i}+x^{2 s+i}$ and one of $2 r-1+i, 2 s+i$ is even and the other odd for all $i$. It follows that all elements $x^{k}$ have the same number of representations as the sum of an odd power and an even power of $x$. Moreover, since $x^{\left(p^{l}-1\right) 2}=-1$, and $\left(p^{l}-1\right) / 2$ is even by hypothesis, -1 belongs to $C_{c}$ and hence the negative of

Received by the editors June 19, 1961. 
every element of $C_{6}$ belongs to $C_{b}$ so that $C_{0} C_{b}$ does not contain the element 0 . It follows that

$$
C_{0} C_{\bullet}=a\left(C_{\bullet}+C_{\bullet}\right) \text {, }
$$

where $a$ denotes the number of times each power of $x$ occurs in $C_{0} C_{0}$. Equating the number of terms on either side we readily get

$$
a=\frac{p^{l}-1}{4}
$$

so that

$$
C_{0} C_{0}=\frac{p^{l}-1}{4}\left(C_{0}+C_{0}\right) .
$$

Let us now observe that

$$
C_{0} F=C_{0} F=\frac{p^{l}-1}{2} F .
$$

Subtracting (2.3) from (2.4) we get

$$
\begin{aligned}
C_{0}^{2}+C_{0} & =\frac{p^{l}-1}{2} C+\frac{p^{l}-1}{4}\left(C_{0}+C_{0}\right) \\
& =C_{0}^{2}+C_{0},
\end{aligned}
$$

which completes the proof of the lemma.

LEMMA 2. If $p^{l} \equiv-1(\bmod 4)$, then

$$
\begin{aligned}
C_{0} C_{0} & =\frac{p^{l}-1}{2} C+\frac{p^{l}-3}{4}\left(C_{0}+C_{0}\right), \\
C_{0}^{2} & =\frac{p^{l}-3}{4} C_{0}+\frac{p^{l}+1}{4} C_{0}, \\
C_{0}^{2} & =\frac{p^{l}+1}{4} C_{0}+\frac{p^{l}-3}{4} C_{0 .} .
\end{aligned}
$$

Proof. The proof is exactly as in Lemma 1 except that in this case -1 is in $C_{0}$ so that $C_{0}$ consists of the negative of the elements of $C_{0}$ and hence

$$
C_{0} C_{\bullet}=\frac{p^{l}-1}{2} C+a\left(C_{\circ}+C_{\bullet}\right)
$$


where $a$ is determined by equating the number of terms on either side.

3. We are now in a position to construct the designs in question, or equivalently to construct difference sets with the parameters (1.1).

Theorem 1. Let $2 m+1=p^{r}, 2 m-1=q^{s}$ where $p, q$ are primes. Let $F_{p}, F_{q}$ be the additive groups of the Galois fields $G F\left(p^{r}\right), G F\left(q^{s}\right)$ respectively and let $G=\left(F_{p}, F_{q}\right)$ be the direct-product of $F_{p}, F_{q}$. Let $C_{p}, C_{p, o}$, $C_{p, e} ; C_{q}, C_{q, o}, C_{q, e}$ denote the sets consisting of the zero element only, the odd powers of the primitive element and the even powers of the primitive element respectively of $F_{p} ; F_{q}$. Then the set $S$ consisting of the pairs

$$
\left(C_{p}+C_{p, o}, C_{q, o}\right), \quad\left(C_{p}+C_{p, e}, C_{q, e}\right), \quad\left(C_{p}, C_{q}\right)
$$

is a difference set in $G$.

Proof. Let us write

$$
\begin{aligned}
& A=\left(C_{p}+C_{p, o}, C_{q, o}\right), \\
& B=\left(C_{p}+C_{p, e}, C_{q, e}\right), \\
& C=\left(C_{p}, C_{q}\right),
\end{aligned}
$$

so that

$$
S=A+B+C .
$$

We shall consider the two cases $p^{r} \equiv \pm 1(\bmod 4)$ separately.

CAse (i). Let $p^{r} \equiv 1(\bmod 4)$. Then $q^{s}=p^{r}-2 \equiv-1(\bmod 4)$. Hence

$$
\begin{aligned}
A A^{-1} & =\left(C_{p}+C_{p, o}, C_{q, o}\right)\left(C_{p}+C_{p, o}, C_{q, e}\right) \\
& =\left(\left(C_{p}+C_{p, o}\right)^{2}, C_{q, o} C_{q, e}\right), \\
B B^{-1} & =\left(C_{p}+C_{p, e}, C_{q, e}\right)\left(C_{p}+C_{p, e}, C_{q, o}\right) \\
& =\left(\left(C_{p}+C_{p, e}\right)^{2}, C_{q, o} C_{q, e}\right), \\
A B^{-1} & =\left(C_{p}+C_{p, o}, C_{q, o}\right)\left(C_{p}+C_{p, e}, C_{q, o}\right) \\
& =\left(\left(C_{p}+C_{p, o}\right)\left(C_{p}+C_{p, e}\right), C_{q, o}^{2}\right), \\
B A^{-1} & =\left(C_{p}+C_{p, e}, C_{q, e}\right)\left(C_{p}+C_{p, o}, C_{q, e}\right) \\
& =\left(\left(C_{p}+C_{p, e}\right)\left(C_{p}+C_{p, o}\right), C_{q, e}^{2}\right) .
\end{aligned}
$$

Hence

$$
\begin{aligned}
S S^{-1}= & \left(\left(C_{p}+C_{p, o}\right)^{2}+\left(C_{p}+C_{p, e}\right)^{2}, C_{q, o} C_{q, e}\right) \\
& +\left(\left(C_{p}+C_{p, o}\right)\left(C_{p}+C_{p, e}\right), C_{q, o}^{2}+C_{q, e}^{2}\right) \\
& +A+B+A^{-1}+B^{-1}+C .
\end{aligned}
$$

Since $p^{r} \equiv 1(\bmod 4)$ we have, by Lemma 1 , 
1962]

$$
\begin{aligned}
\left(C_{p}\right. & \left.+C_{p, o}\right)^{2}+\left(C_{p}+C_{p, e}\right)^{2} \\
& =2\left(C_{p}+C_{p, o}+C_{p, e}\right)+C_{p .0}^{2}+C_{p, e}^{2} \\
& =2 F_{p}+\left(p^{r}-1\right) C_{p}+\frac{p^{r}-3}{2}\left(C_{p, o}+C_{p, e}\right) \\
& =\left(\frac{p^{r}+1}{2}\right)\left(C_{p}+F_{p}\right)=(m+1)\left(C_{p}+F_{p}\right)
\end{aligned}
$$

and

$$
\begin{aligned}
\left(C_{p}\right. & \left.+C_{p, o}\right)\left(C_{p}+C_{p, \mathrm{e}}\right) \\
& =C_{p}+C_{p, \mathrm{o}}+C_{p, \mathrm{e}}+C_{p, 0} C_{p, \mathrm{e}} \\
& =\left(\frac{p^{r}+3}{4}\right) F_{p}-\left(\frac{p^{r}-1}{4}\right) C_{p}=\frac{(m+2)}{2} F_{p}-\frac{m}{2} C_{p}
\end{aligned}
$$

and since $q^{e} \equiv-1(\bmod 4)$ we have, by Lemma 2 ,

$$
\begin{aligned}
C_{q, o} C_{q, o} & =\left(\frac{q^{s}-1}{2}\right) C+\left(\frac{q^{s}-3}{4}\right)\left(C_{q, o}+C_{q, o}\right) \\
& =\left(\frac{q^{s}+1}{4}\right) C+\left(\frac{q^{s}-3}{4}\right) F_{q}=\frac{m}{2} C_{q}+\frac{m-2}{2} F_{q}
\end{aligned}
$$

and

$$
\begin{aligned}
C_{q, 0}^{2}+C_{q, e}^{2} & =\left(\frac{q^{s}-1}{2}\right)\left(C_{q, 0}+C_{q, e}\right) \\
& =\left(\frac{q^{s}-1}{2}\right)\left(F_{q}-C_{q}\right)=(m-1)\left(F_{q}-C_{q}\right) .
\end{aligned}
$$

Moreover,

$$
\begin{aligned}
A+A^{-1}= & \left(C_{p}+C_{p, o}, C_{q, o}\right)+\left(C_{p}+C_{p, o}, C_{q, e}\right) \\
= & \left(C_{p}+C_{p, o}, C_{q, o}+C_{q, e}\right) \\
B+B^{-1}= & \left(C_{p}+C_{p, e}, C_{q, e}\right)+\left(C_{p}+C_{p, e}, C_{q, o}\right) \\
& +\left(C_{p}+C_{p, e,}, C_{q, o}+C_{q, e}\right)
\end{aligned}
$$

so that

$$
A+A^{-1}+B+B^{-1}=\left(C_{p}+F_{p}, F_{q}-C_{q}\right) .
$$

Hence we have 


$$
\begin{aligned}
S S^{-1}= & \left((m+1)\left(C_{p}+F_{p}\right), \frac{m}{2} C_{q}+\frac{m-2}{2} F_{q}\right) \\
& +\left(\frac{(m+2)}{2} F-\frac{m}{2} C_{p},(m-1)\left(F_{q}-C_{q}\right)\right) \\
& +\left(C_{p}+F_{p}, F_{q}-C_{q}\right)+\left(C_{p}, C_{q}\right) \\
= & \left\{\frac{m(m+1)}{2}+\frac{m(m-1)}{2}\right\}\left(C_{p}, C_{q}\right) \\
& +\left\{\frac{(m+1)(m-2)}{2}+\frac{(m+2)(m-1)}{2}+1\right\}\left(F_{p}, F_{q}\right) \\
= & m^{2}\left(C_{p}, C_{q}\right)+\left(m^{2}-1\right) G,
\end{aligned}
$$

which proves that $S$ is a difference set in $G$. Its parameters are easily seen to be

$$
v=4 m^{2}-1, \quad k=2 m^{2}-1, \quad \lambda=m^{2}-1, \quad n=m^{2} .
$$

CASE (ii). Let $p^{r} \equiv-1(\bmod 4), q^{s}=p^{r}-2 \equiv 1(\bmod 4)$.

In this case

$$
\begin{aligned}
A A^{-1} & =\left(C_{p}+C_{p, o}, C_{q, o}\right)\left(C_{p}+C_{p, e}, C_{q, o}\right) \\
& \left.=\left(C_{p}+C_{p, o}\right)\left(C_{p}+C_{p, e}\right), C_{q, o}^{2}\right), \\
B B^{-1} & =\left(C_{p}+C_{p, e}, C_{q, e}\right)\left(C_{p}+C_{p, o}, C_{q, e}\right) \\
& =\left(\left(C_{p}+C_{p, e}\right)\left(C_{p}+C_{p, o}\right), C_{q, e}^{2}\right), \\
A B^{-1} & =\left(C_{p}+C_{p, o}, C_{q, o}\right)\left(C_{p}+C_{p, o}, C_{q, o}\right) \\
& =\left(\left(C_{p}+C_{p, o}\right)^{2}, C_{q, o} C_{q, e}\right) \\
A^{-1} B & =\left(C_{p}+C_{p, e}, C_{q, o}\right)\left(C_{p}+C_{p, e}, C_{q, e}\right) \\
& =\left(\left(C_{p}+C_{p, e}\right)^{2} C_{q, o} C_{q, e}\right) \\
A+A^{-1} & =\left(C_{p}+C_{p, o}, C_{q, o}\right)+\left(C_{p}+C_{p, e}, C_{q, o}\right) \\
& =\left(C_{p}+F_{p,}, C_{q, o}\right), \\
B+B^{-1} & =\left(C_{p}+C_{p, e}, C_{q, e}\right)+\left(C_{p}+C_{p, o}, C_{q, e}\right) \\
& =\left(C_{p}+F_{p,}, C_{q, e}\right) .
\end{aligned}
$$

Hence we have

$$
\begin{aligned}
S S^{-1}= & \left(\left(C_{p}+C_{p, o}\right)\left(C_{p}+C_{p, e}\right), C_{q, o}^{2}+C_{q, e}^{2}\right) \\
& +\left(\left(C_{p}+C_{p, o}\right)^{2}+\left(C_{p}+C_{p, o}\right)^{2}, C_{q, o} C_{q, e}\right) \\
& +\left(C_{p}+F_{p}, F_{q}-C_{q}\right)+\left(C_{p}, C_{q}\right) .
\end{aligned}
$$


But, by Lemma 1,

$$
\begin{aligned}
& C_{q, 0}^{2}+C_{q, e}^{2}=\left(q^{e}-1\right) C_{q}+\frac{q^{e}-3}{2}\left(C_{q, 0}+C_{q, 0}\right) \\
& =\frac{\left(q^{*}+1\right)}{2} C_{q}+\frac{q^{*}-3}{2} F_{q}=m C_{q}+(m-2) F_{q}, \\
& C_{q, 0} C_{q, e}=\frac{q^{2}-1}{4}\left(C_{q, \odot}+C_{q, e}\right)=\frac{m-1}{2}\left(F_{q}+C_{q}\right)
\end{aligned}
$$

and, by Lemma 2,

$$
\begin{aligned}
\left(C_{p}\right. & \left.+C_{p, o}\right)\left(C_{p}+C_{p, e}\right) \\
& =C_{p}+C_{p, 0}+C_{p, e}+C_{p, 0} C_{p, e} \\
& =F_{p}+\frac{p^{r}-1}{2} C_{p}+\frac{p^{r}-3}{4}\left(C_{p, 0}+C_{p, e}\right) \\
& =\frac{p^{r}+1}{4}\left(F_{p}+C_{p}\right)=\frac{m+1}{2}\left(F_{p}-C_{p}\right) \\
\left(C_{p}+\right. & \left.C_{p, o}\right)^{2}+\left(C_{p}+C_{p, e}\right)^{2} \\
& =2\left(C_{p}+C_{p, 0}+C_{p, e}\right)+C_{p, 0}^{2}+C_{p, e}^{2} \\
& =2 F_{p}+\frac{p^{r}-1}{2}\left(C_{p, 0}+C_{p, e}\right) \\
& =\frac{p^{r}+3}{2} F_{p}-\frac{p^{r}-1}{2} C_{p}=(m+2) F_{p}-m C_{p .} .
\end{aligned}
$$

It follows that

$$
\begin{aligned}
S S^{-1}= & \frac{m+1}{2}\left(F_{p}+C_{p}\right), m C_{q}+(m-2) F_{q} \\
& +\left((m+2) F_{p}-m C_{p}, \frac{m-1}{2}\left(F_{q}-C_{q}\right)\right) \\
& +\left(C_{p}+F_{p}, F_{q}-C_{q}\right)+\left(C_{p}, C_{q}\right) \\
= & \left(\frac{m(m+1)}{2}+\frac{m(m-1)}{2}\right)\left(C_{p}, C_{q}\right) \\
& +\left\{\frac{(m+1)(m-2)}{2}+\frac{(m+2)(m-1)}{2}+1\right\}\left(F_{p}, F_{q}\right) \\
= & m^{2}\left(C_{p}, C_{q}\right)+\left(m^{2}-1\right) G
\end{aligned}
$$


which again shows that $S$ is a difference set in $G$ with the parameters

$$
v=4 m^{2}-1, \quad k=2 m^{2}-1, \quad \lambda=m^{2}-1, \quad n=m^{2}
$$

thereby completing the proof of the theorem.

4. A simpler description of the difference set is possible in the case where $2 m+1$ and $2 m-1$ are both primes. We have then the following

THEOREM 2. If two consecutive odd numbers $p, q$ are primes, then the set $S$ consisting of

(i) the prime residue classes $z(\bmod p q)$ such that

$$
\left(\frac{z}{p q}\right)=1 \text {, }
$$

where

$$
\left(\frac{a}{b}\right)
$$

is the Jacobi symbol, and

(ii) the residue classes $p z(\bmod p q)$, if $p \equiv 1(\bmod 4)$, or the residue classes $q z(\bmod p q)$ if $q=1(\bmod 4)$, is a difference set in the additive group of residues $(\bmod p q)$, having the parameters

$$
v=4 m^{2}-1, k=2 m^{2}-1, \lambda=m^{2}-1, n=m^{2} .
$$

Proof. Any prime residue class $z(\bmod p q)$ can be written in the form

$$
z=p x+q y
$$

where $x$ and $y$ are prime residue classes $(\bmod q)$ and $(\bmod p)$ respectively. Now

$$
\begin{aligned}
\left(\frac{z}{p q}\right) & =\left(\frac{p x+q y}{p}\right)\left(\frac{p x+q y}{q}\right) \\
& =\left(\frac{q y}{p}\right)\left(\frac{p x}{q}\right)=\left(\frac{q}{p}\right)\left(\frac{p}{q}\right)\left(\frac{y}{p}\right)\left(\frac{x}{q}\right)=\left(\frac{x}{q}\right)\left(\frac{y}{p}\right),
\end{aligned}
$$

since one of $p, q$ is of the form $4 l+1$. Hence

$$
\left(\frac{z}{p q}\right)=1
$$

if and only if either both $x, y$ are quadratic residues or both nonresidues $\bmod q, \bmod p$ respectively. 
It follows that in the decomposition of the additive group of residues $z(\bmod p q)$ into the direct product of the additive groups of residues $(\bmod q)$ and $(\bmod p)$ respectively defined by

$$
z \rightarrow(y, x)
$$

the set of residues $z(\bmod p q)$ for which

$$
\left(\frac{z}{p q}\right)=1
$$

goes into the union of the sets

$$
\left(C_{p, o}, C_{q, o}\right),\left(C_{p, e}, C_{q, e}\right)
$$

where $C_{p, e}, C_{p, o}$ are the sets of quadratic residues, nonresidues, respectively of $p$ and $C_{q, e}, C_{q, o}$ are the sets of quadratic residues, nonresidues respectively of $q$.

Under the correspondence $p z(\bmod p q)$ goes into $(0, z)$ :

$$
p z \rightarrow(0, z)
$$

and $q z(\bmod p q)$ goes into $(z, 0)$ :

$$
q z \rightarrow(z, 0) \text {. }
$$

It follows that the whole set of residues $p z(\bmod p q)$ corresponds to the set

$$
\left(C_{p}, F_{q}\right)
$$

and the set of residues $q z(\bmod p)$ corresponds to the set

$$
\left(F_{p}, C_{q}\right)
$$

where $F_{p}, F_{q}$ are the additive groups of residues $\bmod p, \bmod q$ respectively and $C_{p}, C_{q}$ are their subsets consisting of their zeros only. Thus the set $S$ in the theorem corresponds to the union of

$$
\left(C_{p}+C_{p, o}, C_{q, o}\right), \quad\left(C_{p}+C_{p, e}, C_{q, e}\right), \quad\left(C_{p}, C_{q}\right)
$$

in case $p \equiv 1(\bmod 4)$, and to the union of

$$
\left(C_{p, o}, C_{q}+C_{q, o}\right), \quad\left(C_{p, e}, C_{q}+C_{q, e}\right), \quad\left(C_{p}, C_{q}\right)
$$

in case $q \equiv 1(\bmod 4)$ which is, in either case, a difference set in the group $\left(F_{p}, F_{q}\right)$, by Theorem 1 . The set $S$ is therefore itself a difference set in the additive group of residues $(\bmod p q)$.

New Delhi, India 